\title{
Are gluten-free foods healthier than non-gluten-free foods? An evaluation of supermarket products in Australia
}

\author{
Jason H. Y. Wu ${ }^{1 *}$, Bruce Neal ${ }^{1,2,3}$, Helen Trevena ${ }^{1}$, Michelle Crino ${ }^{1}$, Wendy Stuart-Smith ${ }^{4}$, \\ Kim Faulkner-Hogg ${ }^{5}$, Jimmy Chun Yu Louie ${ }^{4,6}$ and Elizabeth Dunford ${ }^{1}$ \\ ${ }^{1}$ The George Institute for Global Health, Sydney Medical School, University of Sydney, Level 10, King George V Building, \\ 83-117 Missenden Road, Camperdown, Sydney, NSW 2050, Australia \\ ${ }^{2}$ The School of Public Health, Faculty of Medicine, Epidemiology and Biostatistics, Imperial College of Science, \\ Technology and Medicine, Praed Street, Norfolk Place, London W2 1PG, UK \\ ${ }^{3}$ The Royal Prince Alfred Hospital, Camperdown, NSW 2050, Australia \\ ${ }^{4}$ Discipline of Nutrition and Metabolism, School of Molecular Bioscience, University of Sydney, New South Wales, Australia \\ ${ }^{5}$ Allergy Unit, Royal Prince Alfred Hospital, Camperdown and Food4me Private Practice, New South Wales, Australia \\ ${ }^{6}$ School of Molecular Bioscience and Boden Institute of Obesity, Nutrition, Exercise and Eating Disorders, University of \\ Sydney, New South Wales, Australia
}

(Submitted 11 January 2015 - Final revision received 18 April 2015 - Accepted 13 May 2015 - First published online 29 June 2015)

\begin{abstract}
Despite tremendous growth in the consumption of gluten-free (GF) foods, there is a lack of evaluation of their nutritional profile and how they compare with non-GF foods. The present study evaluated the nutritional quality of GF and non-GF foods in core food groups, and a wide range of discretionary products in Australian supermarkets. Nutritional information on the Nutrition Information Panel was systematically obtained from all packaged foods at four large supermarkets in Sydney, Australia in 2013. Food products were classified as GF if a GF declaration appeared anywhere on the product packaging, or non-GF if they contained gluten, wheat, rye, triticale, barley, oats or spelt. The primary outcome was the 'Health Star Rating' (HSR: lowest score 0.5; optimal score 5), a nutrient profiling scheme endorsed by the Australian Government. Differences in the content of individual nutrients were explored in secondary analyses. A total of 3213 food products across ten food categories were included. On average, GF plain dry pasta scored nearly 0.5 stars less $(P<0 \cdot 001)$ compared with non-GF products; however, there were no significant differences in the mean HSR for breads or ready-to-eat breakfast cereals $(P \geq 0 \cdot 42$ for both). Relative to non-GF foods, GF products had consistently lower average protein content across all the three core food groups, in particular for pasta and breads ( 52 and $32 \%$ less, $P<0.001$ for both). A substantial proportion of foods in discretionary categories carried GF labels (e.g. $87 \%$ of processed meats), and the average HSR of GF discretionary foods were not systematically superior to those of non-GF products. The consumption of GF products is unlikely to confer health benefits, unless there is clear evidence of gluten intolerance.
\end{abstract}

Key words: Gluten: Nutrient profiling: Food labels

Health effects of gluten have received increasing attention both in medical research and popular media, and remain highly controversial ${ }^{(1-3)}$. People with diagnosed coeliac disease require a lifelong strictly gluten-free $(\mathrm{GF})$ diet $^{(4)}$. In addition to coeliac disease patients, it has been hypothesised that a substantial proportion of the population may be gluten intolerant (non-coeliac gluten sensitivity), and could benefit from reducing gluten in their diet ${ }^{(5)}$. However, clinical evidence for the existence of such conditions and other purported adverse health effects of gluten remain inconsistent ${ }^{(6)}$. Nevertheless, there is growing popular perception that GF foods are healthier, and in recent years, there has been a dramatic increase in demand and consumption of GF foods in many Western countries. For example, nearly one-third of adults in the USA have expressed interest in avoiding or cutting down on gluten in their diets, and sales of GF foods reached approximately $\$ 10$ billion in 2013 , with most of the increased demand for GF foods from those without clinically diagnosed coeliac disease ${ }^{(3,7)}$. A rapid growth in the sale of GF foods has also been observed in the $\mathrm{UK}^{(8)}$.

Despite the tremendous rise in popularity and consumption of GF foods, there is a lack of evaluation of their nutritional profile and how they compare with non-GF foods. Such an assessment is important for several reasons. Gluten-containing

Abbreviations: GF, gluten-free; HSR, Health Star Rating; NIP, Nutrition Information Panel; RTE, ready-to-eat.

*Corresponding author: J. H. Y. Wu, fax +6129993 4501, email jwu1@georgeinstitute.org.au 
grains such as wheat, rye and barley are important sources of nutrients. Staple foods that traditionally contain these grains are core to the diet of many countries, and consumed by large proportions of the population ${ }^{(9)}$. There are concerns that removal or substitution of these grains from GF products with other ingredients could adversely affect nutrient intake in those consuming a GF diet ${ }^{(10,11)}$. Furthermore, consumers may perceive GF products as healthier than non-GF foods, and food companies may market them as such and charge a premium price ${ }^{(12,13)}$. This may occur even when the foods concerned are energy-rich, nutrient-poor discretionary products such as cakes and biscuits ${ }^{(12-14)}$. For most food categories, it is unclear whether GF products contain comparable, higher or lower levels of sugar, salt and saturated fat relative to non-GF products.

To address these gaps in knowledge, we conducted an evaluation of the nutritional quality of GF foods in core food groups and a wide range of discretionary product categories available in Australian supermarkets in 2013, and compared their nutritional profile with non-GF products.

\section{Methods \\ Design and data}

Nutritional information for each food product was obtained from the Nutrition Information Panel (NIP), and data were collected using previously described methods ${ }^{(15,16)}$. Briefly, between July and December 2013, NIP data were systematically obtained from all packaged food products available for sale at four large supermarket stores (Coles, Woolworths, ALDI and IGA) in Sydney, New South Wales, Australia. Where exactly the same product was for sale in more than one supermarket, it was recorded only once. Where the same product was presented in different pack sizes, only one entry was recorded. For each food product, the manufacturer, brand and product name, nutrient content per $100 \mathrm{~g}$ as appeared on the NIP, and gluten status were recorded (see below). Data were entered into The George Institute's branded food composition database according to standardised procedures ${ }^{(17)}$. Likewise, data were verified according to a defined quality assurance protocol and workflow, which includes screening for outliers and missing values, checking data entry accuracy by two study personnel independently, and resolving queries and discrepancies by review of the original NIP data, consultation between the research personnel, review of the manufacturer's website, or follow-up with the manufacturer directly.

\section{Product categories included}

The staple food categories that we included were those typically containing gluten and comprised breads, ready-to-eat (RTE) breakfast cereals and dry plain pasta. These are important contributors to energy intake in typical Western diets including Australia, and are part of the core foods recommended by the Australian Dietary Guidelines ${ }^{(9,18)}$. Likewise, we evaluated multiple categories of discretionary foods including those that utilise large quantities of gluten-containing grains (cereal bars, cake mixes/cakes and sweet biscuits) as well as others that typically include smaller quantities and for which GF alternatives are often available (ice cream, corn and potato chips, cured meats, sausages and hot dogs, and sugar-based confectioneries). Food categories were also selected if they contained at least twenty GF and twenty non-GF products to allow meaningful comparison and statistical inference between the nutritional composition of GF and non-GF foods.

\section{Determination of gluten status}

A product was classified as GF if a GF declaration appeared on the NIP, or anywhere else on the product packaging. A product was coded as non-GF if it contained any of the following ingredients: gluten; wheat; rye; triticale; barley; oats; or spelt. Glucose syrup derived from wheat was considered to be a GF ingredient. We excluded from analyses the food products that did not carry a GF label, but were determined to be likely GF based on inspection of the ingredient list.

\section{Outcomes}

The primary outcome used to compare the nutritional quality of GF $v$. non-GF products was the 'Health Star Rating' (HSR) system, a voluntary nutrient profiling scheme endorsed by the Australian Government with the aim of helping consumers to choose healthier foods ${ }^{(19)}$. The HSR system rates a product between 0.5 and 5 stars (increasing in 1/2-star increments, with more stars indicating higher nutritional quality). The number of stars for a food product is calculated based on an algorithm that takes into account the quantity of specific food components and the estimated overall healthiness of the product. Details of the HSR algorithm are provided in the Supplementary material (available online). In secondary analyses, we also explored differences in the content of energy (kJ), saturated fat, total sugars, $\mathrm{Na}$, protein and dietary fibre per $100 \mathrm{~g}$ between GF and non-GF products. Australian law does not require mandatory labelling of fibre content as part of the NIP, so we restricted the analysis of fibre to only those food products with available information.

\section{Statistical analyses}

Data are presented as means and standard deviations. Summary data for energy and nutrients were presented per $100 \mathrm{~g}$ of each food. Differences in mean HSR and nutrient content between GF and non-GF products were assessed by linear regression analysis. A two-sided $P$ value of $<0.05$ was considered as statistically significant. Statistical analyses were conducted using Stata 13.1 (Stata Corporation).

\section{Results}

A total of 3213 food products across ten food categories were included in the analyses. The top three manufacturers of GF and non-GF products and the number of products in each 
category are provided in online Supplementary Table S1. The number of products ranged from 154 for corn and potato chip products to 550 for sweet biscuits. In almost all of the food categories, the majority of products were non-GF (percentage of GF products 10-36\%), with the only exception being cured meats, sausages and hot dogs for which most ( $87 \%$ ) of the products were labelled as 'GF'. For non-GF products, the top three manufacturers were mainly large international food manufacturers (e.g. Kellogg and Mondelez) or major Australian supermarket retailers selling their own brand (e.g. Woolworths and Coles). Conversely, GF products came from a more diverse group of manufacturers, and the leading companies were mostly different across product categories.

The mean HSR and nutrient content of GF $v$. non-GF products in the core food groups are summarised in Table 1. On average, GF plain dry pasta scored nearly 0.5 stars less (95\% CI $-0.38,0.57, P<0.001)$ compared with non-GF products; however, there were no significant differences in the mean HSR for breads or RTE breakfast cereals $(P \geq 0.42$ for both). Relative to non-GF foods, GF products had consistently lower average protein content across all the three core food groups, in particular for plain dry pasta and breads (52 and $32 \%$ less, $P<0.001$ for both). The contents of total energy, $\mathrm{Na}$, saturated fat and total sugars were similar between GF and non-GF core foods. While GF breads had significantly higher mean dietary fibre content $(2 \cdot 2 \mathrm{~g} / 100 \mathrm{~g}, P<0 \cdot 001)$, the opposite was true for RTE breakfast cereals (Fig. 1).

HSR were similar for four out of the seven discretionary food groups assessed, including cereal bars, cake mixes/ cakes, sweet biscuits, and cured meats, sausages and hot dogs (Table 2). Consistent with this observation, there were no clear patterns of differences in the content of individual nutrients between GF and non-GF products in these food categories (Table 2 and see online Supplementary Fig. S1). These foods were generally high in $\mathrm{Na}$, saturated fat and sugar content and often exhibited reciprocal changes in the levels of these nutrients. For example, while GF cake mixes and cakes had lower saturated fat levels compared with non-GF products (by $2 \cdot 1 \mathrm{~g} / 100 \mathrm{~g}, P=0.001$ ), they had higher total sugar levels (by $8.1 \mathrm{~g} / 100 \mathrm{~g}, P<0.001$ ), resulting in similar mean total energy $(1469$ v. $1501 \mathrm{~kJ} / 100 \mathrm{~g})$. In the remaining three discretionary food categories examined (ice cream, corn and potato chips, and sugar-based confectioneries), GF products had significantly higher HSR (mean difference $0 \cdot 2-0 \cdot 8$ stars, $P \leq 0 \cdot 01$ for both), which were largely driven by lower mean levels of saturated fat (ice cream and corn and potato chips) or total sugars (ice cream and sugar-based confectioneries) and therefore energy density (all the three product categories).

\section{Discussion}

Based on a large cross-sectional survey, our data show that GF products in the core food categories had overall similar nutritional profiles compared with non-GF products, with the notable exception being that GF products had lower average protein levels. Furthermore, while GF products had slightly

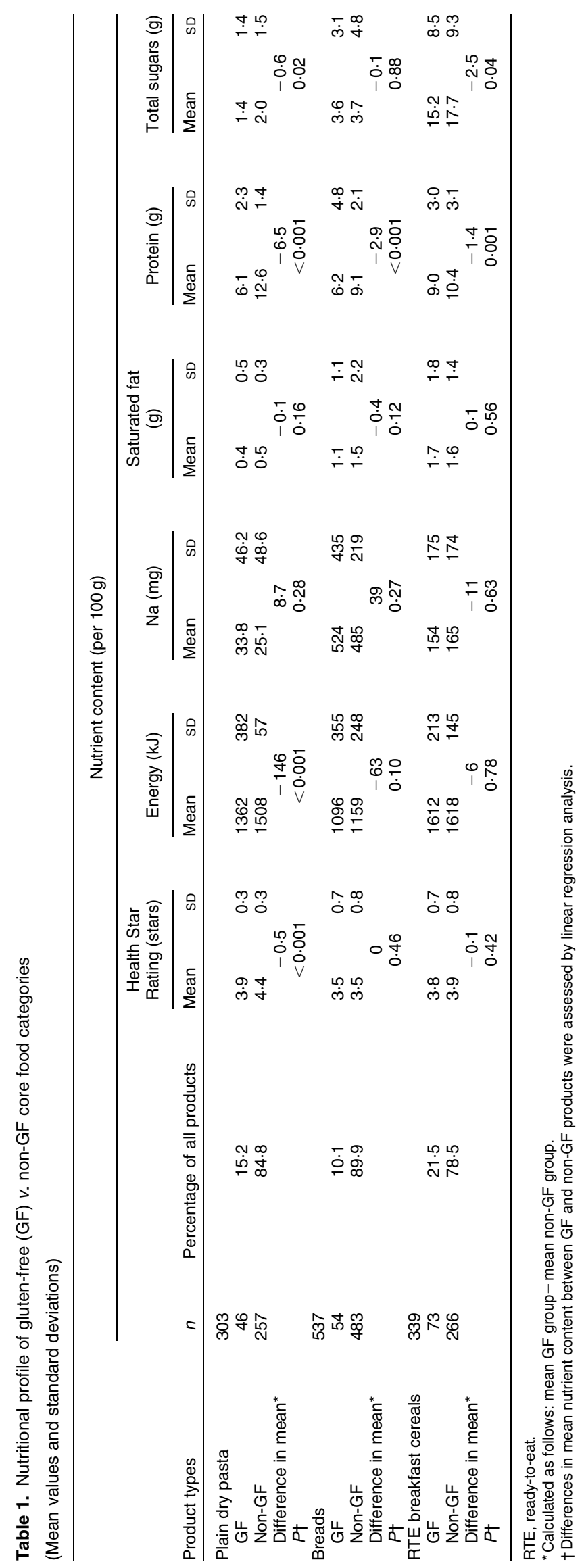


better average nutritional profiles compared with their non-GF counterparts in several discretionary food categories, the overall quality of these products remained poor with typically high levels of sugar, saturated fat and salt.

Gluten is an important part of traditional cereal-based foods such as bread and pasta because it confers desirable functional (e.g. strengthening the structure of bread) and sensory (e.g. improved mouthfeel) characteristics important to food manufacturers and consumers ${ }^{(20)}$. To compensate for the absence of gluten, GF products are developed using a wide variety and mixture of GF flour, fibre, hydrocolloids and enzymes ${ }^{(21)}$, and concerns have been raised regarding the nutritional profile of GF products. For example, prior surveys of a limited number of GF products have suggested frequent dependence on substitute ingredients with low fibre content ${ }^{(22,23)}$. It has also been speculated that GF products may contain more sugar and fat to improve palatability, with the concern that this could result in higher energy intake and weight gain ${ }^{(24)}$. A key finding from our analyses of nearly 1200 core foods is the lower average protein content of GF products, which suggests that carbohydrate-rich, but protein-poor ingredients such as maize starch, white rice flour, potato starch or tapioca starch are likely to have been used as substitutes in these food categories. As such ingredients are often also very low in vitamins and minerals, our findings highlight the need for future studies to examine the levels of these nutrients in GF core foods. The present results do not support the contention that GF core foods are consistently lower in fibre content or that the nutritional quality of GF foods is seriously adversely influenced by the addition of saturated fat or sugar.

Breads, RTE breakfast cereals and pasta are important sources of nutrients in the Australian population, with the most recent Australian Health Survey reporting that approximately two-thirds and one-third of the population consumed breads and RTE breakfast cereals on the day of assessment ${ }^{(9)}$. The differences in protein content between GF and non-GF core foods that we identified have also been reported by others $^{(25)}$. However, this difference is unlikely to have a

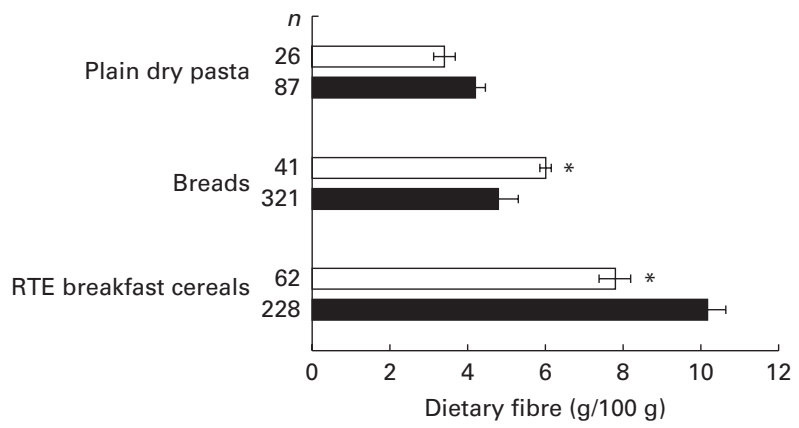

Fig. 1. Dietary fibre levels of gluten-free $(G F, \square)$ and non-gluten-free (non-GF, 口) pasta, breads and ready-to-eat (RTE) breakfast cereal products. Products were identified from four major supermarkets in Sydney, Australia in 2013, and analyses were restricted to products with dietary fibre information available on their Nutrition Information Panel. Values are means, with their standard errors represented by vertical bars. * Mean value was significantly different from that of non-GF products $(P<0.05$; linear regression). substantial impact on protein intake in those following a GF diet if they also eat meat, dairy and egg products. Nevertheless, those who do not (e.g. vegans) could be particularly affected, and education regarding alternative protein sources (e.g. legumes and nuts) is important for this group.

There has been a dramatic increase in the demand for GF products over the last several years, driven in part by a perception that these products are healthier than their non-GF counterparts ${ }^{(2,3)}$. A large number of discretionary products that do not naturally contain gluten (e.g. processed meats and corn and potato chips) and that now carry GF labelling suggests that food manufacturers may be employing GF labelling as a tool to market discretionary food items. The average nutritional qualities of GF discretionary foods were not systematically superior to those of non-GF products, although some were better than their gluten-containing counterparts. There is growing evidence that the 'health halo' effect whereby products are labelled as 'healthier' (e.g. low fat) can mislead consumers about elements such as energy content and portion sizes, resulting in increased consumption ${ }^{(26-29)}$. The effects of GF labelling on consumer perception and behaviour is not well established; however, there is a clear risk that consumers could misconstrue GF status as an indicator of healthiness. Another potential risk is that non-coeliac subjects who choose to consume a GF diet may unnecessarily limit the variety and adversely affect the quality of their diet, which could have a substantial influence on health outcomes ${ }^{(30)}$. Therefore, it is also important for future studies to assess the overall dietary pattern (i.e. intake of specific foods such as whole grains, fruits and vegetables) in populations consuming a GF diet.

The present study has several strengths. Nutritional information was collected using standardised methods with rigorous quality control that reduced the likelihood of data errors. We sampled a large number of products that enhanced statistical power to assess differences in nutritional quality between GF and non-GF products. The collection of packaged foods from leading Australian retailers increased the relevance of these findings to Australian consumers. Simultaneous assessment of both core and discretionary food products provided novel insights into the nutritional quality of GF products across diverse food domains that are consumed in significant quantities by a large proportion of the population.

Potential limitations are the absence of 'gold-standard' chemical analysis to assess individual nutrients in the food products and our reliance on the NIP. Prior independent assessments of NIP data suggest that they are generally accurate $^{(31)}$. Our reliance on the NIP also meant that we were unable to systematically evaluate the levels of other nutrients of interest (e.g. folate, thiamin and Fe) that may differ between GF and non-GF products ${ }^{(22,23,32)}$. Dietary fibre information was available for only a subset of the products evaluated, and therefore these results should be interpreted with caution. We did not collect and compare the price of GF and non-GF products; furthermore, as the present study examined packaged foods from large supermarket chains in Australia, the findings may not be generalisable to products found in other types of food retailers or to other countries. 
Table 2. Nutritional profile of gluten-free (GF) v. non-GF discretionary food categories

(Mean values and standard deviations)

\begin{tabular}{|c|c|c|c|c|c|c|c|c|c|c|c|c|c|c|}
\hline \multirow[b]{2}{*}{ Product types } & \multicolumn{2}{|c|}{ Nutrient content (per $100 \mathrm{~g}$ ) } & \multicolumn{2}{|c|}{$\begin{array}{l}\text { Health Star } \\
\text { Rating (stars) }\end{array}$} & \multicolumn{2}{|c|}{ Energy (kJ) } & \multicolumn{2}{|c|}{$\mathrm{Na}(\mathrm{mg})$} & \multicolumn{2}{|c|}{$\begin{array}{l}\text { Saturated fat } \\
\text { (g) }\end{array}$} & \multicolumn{2}{|c|}{ Protein $(\mathrm{g})$} & \multicolumn{2}{|c|}{$\begin{array}{l}\text { Total sugars } \\
\text { (g) }\end{array}$} \\
\hline & $n$ & Percentage of all products & Mean & SD & Mean & SD & Mean & SD & Mean & SD & Mean & SD & Mean & SD \\
\hline Cereal bars & 237 & & & & & & & & & & & & & \\
\hline GF & 60 & $25 \cdot 3$ & $2 \cdot 6$ & 0.9 & 1907 & 299 & 53.9 & $52 \cdot 3$ & $5 \cdot 3$ & 4.6 & $9 \cdot 7$ & 3.8 & $30 \cdot 6$ & $10 \cdot 4$ \\
\hline Non-GF & 177 & 74.7 & $2 \cdot 6$ & 0.9 & 1765 & 224 & 135 & 105 & $6 \cdot 0$ & $4 \cdot 0$ & $8 \cdot 1$ & 3.4 & $25 \cdot 9$ & 7.5 \\
\hline Difference in mean ${ }^{*}$ & & & \multirow{2}{*}{\multicolumn{2}{|c|}{$\begin{array}{c}0 \\
0.75\end{array}$}} & \multirow{2}{*}{\multicolumn{2}{|c|}{$\begin{array}{c}142 \\
<0.001\end{array}$}} & \multicolumn{2}{|c|}{-81} & \multirow{2}{*}{\multicolumn{2}{|c|}{$\begin{array}{l}-0.7 \\
0.31\end{array}$}} & \multirow{2}{*}{\multicolumn{2}{|c|}{$\begin{array}{c}1.6 \\
0.002\end{array}$}} & \multicolumn{2}{|c|}{4.7} \\
\hline$P \dagger$ & & & & & & & \multicolumn{2}{|c|}{$<0.001$} & & & & & \multicolumn{2}{|c|}{$<0.001$} \\
\hline Cake mixes/cakes & 491 & & \multicolumn{2}{|c|}{0.75} & & & & & \multicolumn{2}{|c|}{0.31} & & & & \\
\hline GF & 59 & $12 \cdot 0$ & 1.7 & 0.8 & 1469 & 307 & 300 & 215 & 3.4 & 4.9 & 4.9 & 6.4 & $44 \cdot 1$ & $24 \cdot 1$ \\
\hline Non-GF & 432 & $88 \cdot 0$ & 1.6 & 0.7 & 1501 & 290 & 319 & 200 & 5.5 & $4 \cdot 6$ & $4 \cdot 6$ & $1 \cdot 3$ & $36 \cdot 0$ & 13.5 \\
\hline Difference in mean* & & & \multirow{2}{*}{\multicolumn{2}{|c|}{$\begin{array}{l}0.1 \\
0.65\end{array}$}} & \multirow{2}{*}{\multicolumn{2}{|c|}{$\begin{array}{l}-32 \\
0.43\end{array}$}} & \multirow{2}{*}{\multicolumn{2}{|c|}{$\begin{array}{l}-19 \\
0.50\end{array}$}} & \multirow{2}{*}{\multicolumn{2}{|c|}{$-2 \cdot 1$}} & \multirow{2}{*}{\multicolumn{2}{|c|}{0.3}} & \multirow{2}{*}{\multicolumn{2}{|c|}{$\begin{array}{c}8.1 \\
<0.001\end{array}$}} \\
\hline$P \dagger$ & & & & & & & & & & & & & & \\
\hline Sweet biscuits & 550 & & & & & & & & & & & & & \\
\hline GF & 63 & 11.5 & $1 \cdot 2$ & 0.7 & 1942 & 250 & 183 & 158 & 13.9 & 5.4 & 4.5 & $2 \cdot 1$ & $31 \cdot 3$ & $7 \cdot 8$ \\
\hline Non-GF & 487 & 88.5 & $1 \cdot 1$ & 0.7 & 1978 & 200 & 240 & 127 & 11.7 & $5 \cdot 6$ & $5 \cdot 8$ & 1.5 & 33.4 & $10 \cdot 7$ \\
\hline Difference in mean* & & & & & & & & & 2 & & - & & & \\
\hline$P \dagger$ & & & & & 0 . & & & & 0.0 & & $<0$ & & & \\
\hline Ice cream & 217 & & & & & & & & & & & & & \\
\hline GF & 25 & 11.5 & $2 \cdot 8$ & 0.6 & 728 & 195 & $49 \cdot 1$ & 17.5 & $5 \cdot 1$ & $3 \cdot 8$ & $3 \cdot 3$ & $1 \cdot 3$ & $18 \cdot 4$ & $4 \cdot 6$ \\
\hline Non-GF & 192 & 88.5 & $2 \cdot 0$ & 0.8 & 1073 & 284 & $70 \cdot 4$ & $35 \cdot 7$ & 8.9 & 4.7 & $3 \cdot 6$ & $1 \cdot 1$ & $23 \cdot 6$ & 3.7 \\
\hline Difference in mean* & 政 & 80 & & . & -3 & 5 & - & 3 & -5 & 3 & - & t & - & 2 \\
\hline$P \dagger$ & & & & & $<0$ & & & & $<0$ & & & & & \\
\hline Corn and potato chips & 154 & & & & & & & & & & & & & \\
\hline GF & 56 & $36 \cdot 4$ & 3.5 & 0.7 & 1996 & 186 & 596 & 457 & $4 \cdot 0$ & 3.7 & $6 \cdot 0$ & $2 \cdot 1$ & 3.0 & $2 \cdot 6$ \\
\hline Non-GF & 98 & 63.6 & $2 \cdot 8$ & 0.7 & 2122 & 121 & 645 & 211 & 9.4 & $5 \cdot 3$ & 6.4 & 1.4 & 2.9 & 1.5 \\
\hline Difference in mean* & & & & & - & & & & -5 & & & & & \\
\hline$P \dagger$ & & & & & $<0$ & & & & $<0$ & & & & & \\
\hline Cured meats, sausages and hot dogs & 179 & & & & & & & & & & & & & \\
\hline GF & 156 & $87 \cdot 2$ & 1.4 & 0.9 & 1134 & 352 & 1056 & 496 & 8.5 & 3.7 & $18 \cdot 0$ & 5.5 & 0.9 & 0.5 \\
\hline Non-GF & 23 & $12 \cdot 9$ & 1.4 & 0.8 & 1104 & 301 & 1005 & 347 & $7 \cdot 0$ & $3 \cdot 0$ & $16 \cdot 6$ & 3.9 & 0.9 & $0 \cdot 2$ \\
\hline Difference in mean* & & & 0.1 & & 30 & & 51 & & 1 & & 1 & & & \\
\hline$P+$ & & & 0.87 & & 0.70 & & 0.63 & & 0. & & & & & \\
\hline Sugar-based confectioneries & 206 & & & & & & & & & & & & & \\
\hline GF & 45 & $21 \cdot 8$ & $2 \cdot 0$ & 0.8 & 1380 & 198 & 51.9 & 102 & $2 \cdot 0$ & 3.3 & 3.4 & 2.9 & 38.8 & 31.9 \\
\hline Non-GF & 161 & $78 \cdot 2$ & $1 \cdot 8$ & 0.5 & 1462 & 119 & $86 \cdot 9$ & 96 & $1 \cdot 0$ & 1.0 & $2 \cdot 9$ & 1.7 & $52 \cdot 4$ & 14.5 \\
\hline $\begin{array}{l}\text { Difference in mean* } \\
\text { Pt }\end{array}$ & & & & & - & & & & 1 & & 0 & & & \\
\hline
\end{tabular}

*Calculated as follows: mean GF group - mean non-GF group.

†Differences in mean nutrient content between GF and non-GF products were assessed by linear regression analysis. 
In conclusion, the consumption of GF products is unlikely to confer health benefits, unless there is clear evidence of coeliac disease, gluten intolerance or allergy to gluten-containing grains. There is a moderate likelihood that GF labelling is being used to infer healthiness for discretionary items, which is unwarranted. Given the adverse health effects caused by poor diets in Australia and other parts of the world, policy initiatives should target increased consumption of core foods such as whole grains, fruit and vegetables and reduced consumption of discretionary foods (GF or otherwise) as a public health priority.

\section{Supplementary material}

To view supplementary material for this article, please visit http://dx.doi.org/10.1017/S0007114515002056

\section{Acknowledgements}

B. N. was supported by an Australian Research Council Future Fellowship (DP100100295) and a National Health and Medical Research Council (NHMRC) of Australia Senior Research Fellowship (APP100311). He holds a NHMRC Program Grant (APP1052555). B. N., J. H. Y. W. and M. C. work within a NHMRC Centre for Research Excellence (APP1041020). H. T. was supported by a postgraduate scholarship from the NHMRC. E. D. was supported by a NHMRC Early Career Fellowship.

The authors' contributions are as follows: J. H. Y. W., B. N. and E. D. contributed to the study concept and design; B. N. obtained funding; J. H. Y. W. and E. D. conducted the research, the acquisition of the data, and the statistical analyses; J. H. Y. W. drafted the manuscript and had primary responsibility for the final content. All authors interpreted the data, critically revised the manuscript for important intellectual content, and approved the final manuscript.

There are no conflicts of interest.

\section{References}

1. Leonard MM \& Vasagar B (2014) US perspective on glutenrelated diseases. Clin Exp Gastroenterol 7, 25-37.

2. Berry S (2014) A grain of truth to gluten intolerance. The Sydney Morning Herald, 27 May. http://www. smh.com.au/lifestyle/diet-and-fitness/a-grain-of-truth-togluten-intolerance-20140527-zrpb3.html (accessed February 2015).

3. Strom S (2014) A big bet on gluten-free. New York Times, 17 February. http://www.nytimes.com/2014/02/18/business/ food-industry-wagers-big-on-gluten-free.html?_r=2 (accessed February 2015).

4. Ludvigsson JF, Bai JC, Biagi F, et al. (2014) Diagnosis and management of adult coeliac disease: guidelines from the British Society of Gastroenterology. Gut 63, 1210-1228.

5. Mansueto P, Seidita A, D'Alcamo A, et al. (2014) Non-celiac gluten sensitivity: literature review. J Am Coll Nutr 33, $39-54$.

6. Biesiekierski JR, Muir JG \& Gibson PR (2013) Is gluten a cause of gastrointestinal symptoms in people without celiac disease? Curr Allergy Asthma Rep 13, 631-638.
7. NDP Dieting Monitor (2012) Is gluten-free eating a trend worth noting? http://www.npd.com/perspectives/food-forthought/gluten-free-2012.html (accessed September 2014).

8. Just-food (2013) Gluten free: the UK market. http:// www.just-food.com/management-briefing/the-uk-market_id 123018.aspx (accessed December 2014).

9. Australian Bureau of Statistics (2011-2012) Australian Health Survey: nutrition first results - foods and nutrients. http://www.abs.gov.au/ausstats/abs@.nsf/Lookup/4364.0.55. 007main + features12011-12 (accessed September 2014).

10. Mazzeo T, Cauzzi S, Brighenti F, et al. (2014) The development of a composition database of gluten-free products. Public Health Nutr 18, 1353-1357.

11. Nash DT \& Slutzky AR (2014) Gluten sensitivity: new epidemic or new myth? Every major change in our diet carries with it the possibility of unforeseen risks. Am J Cardiol 114, 1621-1622.

12. Singh J \& Whelan K (2011) Limited availability and higher cost of gluten-free foods. J Hum Nutr Diet 24, 479-486.

13. Stevens L \& Rashid M (2008) Gluten-free and regular foods: a cost comparison. Can J Diet Pract Res 69, 147-150.

14. Lee AR, Ng DL, Zivin J, et al. (2007) Economic burden of a gluten-free diet. J Hum Nutr Diet 20, 423-430.

15. Dunford E, Trevena H, Goodsell C, et al. (2014) FoodSwitch: a mobile phone app to enable consumers to make healthier food choices and crowdsourcing of national food composition data. JMIR Mhealth Uhealth 2, e37.

16. Trevena H, Neal B, Dunford E, et al. (2014) An evaluation of the effects of the Australian food and health dialogue targets on the sodium content of bread, breakfast cereals and processed meats. Nutrients $\mathbf{6}$, 3802-3817.

17. Dunford E, Webster J, Metzler AB, et al. (2012) International collaborative project to compare and monitor the nutritional composition of processed foods. Eur J Prev Cardiol 19, 1326-1332.

18. Australian Dietary Guidelines (2013) National Health and Medical Research Council of Australia. http://www.nhmrc. gov.au/guidelines/publications/n55 (accessed December 2014).

19. Australian Government Department of Health (2014) Legislative and Governance Forum on Food Regulation Final Communique. Canberra: Australian Government. http://www.health.gov.au/internet/main/publishing.nsf/ Content/mr-yr14-dept-dept006.htm (accessed December 2014).

20. Scazzina F, Dall'Asta M, Pellegrini N, et al. (2014) Glycaemic index of some commercial gluten-free foods. Eur J Nutr (Epublication ahead of print version 17 October 2014).

21. Gallagher E, Gormley TR \& Arendt EK (2004) Recent advances in the formulation of gluten-free cereal-based products. Trends Food Sci Technol 15, 143-152.

22. Saturni L, Ferretti G \& Bacchetti T (2010) The gluten-free diet: safety and nutritional quality. Nutrients $\mathbf{2}, 16-34$.

23. Thompson $\mathrm{T}$ (2000) Folate, iron, and dietary fiber contents of the gluten-free diet. J Am Diet Assoc 100, 1389-1396.

24. Cross C (2013) Gluten-free industry is healthy, but is the food? CMAJ 185, E610.

25. Miranda J, Lasa A, Bustamante MA, et al. (2014) Nutritional differences between a gluten-free diet and a diet containing equivalent products with gluten. Plant Foods Hum Nutr 69 , $182-187$.

26. Ebneter DS, Latner JD \& Nigg CR (2013) Is less always more? The effects of low-fat labeling and caloric information on food intake, calorie estimates, taste preference, and health attributions. Appetite 68, 92-97. 
27. Faulkner GP, Pourshahidi LK, Wallace JM, et al. (2014) Perceived 'healthiness' of foods can influence consumers' estimations of energy density and appropriate portion size. Int J Obes (Lond) 38, 106-112.

28. Wansink B \& Chandon P (2006) Can "low-fat" nutrition labels lead to obesity? J Market Res 43, 605-617.

29. Chandon P \& Wansink B (2007) The biasing health halos of fast-food restaurant health claims: lower calorie estimates and higher side-dish consumption intentions. I Consumer Res 34, 301-314
30. Mozaffarian D \& Ludwig DS (2010) Dietary guidelines in the 21 st century - a time for food. JAMA 304, 681-682.

31. Food Standards Australia New Zealand (2009) Sodium levels in a range of packaged and take-away foods. http://www. foodstandards.gov.au/publications/pages/sodiumlevelsina range 4648.aspx (accessed December 2014).

32. Thompson T, Dennis M, Higgins LA, et al. (2005) Gluten-free diet survey: are Americans with coeliac disease consuming recommended amounts of fibre, iron, calcium and grain foods? J Hum Nutr Diet 18, 163-169. 